\title{
Electro-Optic Frequency Response Shaping using Embedded FIR Filters in Slow-Wave Modulators
}

\author{
Laurens Breyne (i), Member, IEEE, Joris Lambrecht $\left.{ }^{(}\right)$, Michiel Verplaetse ${ }^{(0)}$, Member, IEEE, \\ Xin Yin ${ }^{\circledR}$, Senior Member, IEEE, Günther Roelkens ${ }^{\circledR}$, Senior Member, IEEE, Peter Ossieur, Member, IEEE, \\ and Johan Bauwelinck ${ }^{\circledR}$, Senior Member, IEEE
}

\begin{abstract}
A novel method is presented to embed finite-impulseresponse filters in slow-wave Mach-Zehnder modulators. This allows to adjust the electro-optic frequency response to the designer's needs. The filter is embedded by adding optical delay lines and optical crossings between phase shifter segments. The position of the delay lines and crossing in the modulator and the delay line length determine the final response. In this work, we provide a full analysis and modeling approach of the proposed technique and apply it to a silicon photonic modulator. However, the technique is generally applicable to slow-wave modulators and thus not limited to a silicon photonics platform. The modeling is verified using measurements on the manufactured devices. A shaped modulator is used in C-band transmission experiments with $56 \mathrm{~Gb} / \mathrm{s} \mathrm{NRZ}$ data over $3 \mathrm{~km}$ fiber to counteract chromatic dispersion and show the advantage over a standard modulator.
\end{abstract}

Index Terms-High-speed optical modulators, Mach-Zehnder modulators, slow-wave modulators, silicon photonics, FIR filters.

\section{INTRODUCTION}

$\mathbf{T}$ HE ever-increasing growth in network traffic drives both industry and academia to push the capacity of optical interconnects to support astounding datarates. The last decade has proven that integrated photonic systems have a key role to play in this evolution by allowing compact, fast, innovative and cheap

Special Research Fund (BOF) of Ghent University, in part by the Research Foundation-Flanders (FWO), and in part by EU ERC Grant 695495 (ATTO). (Corresponding author: Laurens Breyne.)

Laurens Breyne is with Department of Information Technology (INTEC), IDlab, Ghent University-imec, 9052 Ghent, Belgium, also with Department of Information Technology (INTEC), Photonics Research Group, Ghent University-imec, 9052 Ghent, Belgium, and also holds a Ph.D. Fellowship of the Special Research Fund, Ghent University, 9000 Ghent, Belgium (e-mail: laurens.breyne@ugent.be).

Joris Lambrecht, Xin Yin, Peter Ossieur, and Johan Bauwelinck are with the Department of Information Technology (INTEC), IDlab, Ghent University-imec, 9052 Ghent, Belgium (e-mail: joris.lambrecht@ugent.be; xin.yin@ugent.be; peter.ossieur@ugent.be; johan.bauwelinck@ugent.be).

Michiel Verplaetse is with the Department of Information Technology (INTEC), IDlab, Ghent University-imec, 9052 Ghent, Belgium, and also holds a $\mathrm{Ph} . \mathrm{D}$. Fellowship of Research Foundation-Flanders, 1000 Brussel, Belgium (e-mail: michiel.verplaetse@ugent.be).

Günther Roelkens is with the Department of Information Technology (INTEC), Photonics Research Group, Ghent University-imec, 9052 Ghent, Belgium (e-mail: gunther.roelkens@ugent.be).

Color versions of one or more figures in this article are available at https: //doi.org/10.1109/JLT.2020.3043131.

Digital Object Identifier 10.1109/JLT.2020.3043131 devices to be manufactured in large volumes. The devices can be used both in externally intensity-modulated/direct-detection (IM/DD) as well as coherent communication systems. Especially silicon photonic systems gained a lot of interest. Thanks to the large refractive index difference between the silicon core and silicon dioxide cladding, compact, low-loss structures are possible. The compatibility with CMOS manufacturing lines provides high-volume, high-yield and low-cost production while also allowing easy integration with e.g. (Bi)CMOS driver circuits.

Three types of silicon optical modulators have gained particular interest: Mach-Zehnder modulators (MZMs) [1]-[10], electro-absorption modulators (EAMs) [11], [12] and microring modulators (MRMs) [13]. EAMs and MRMs offer a very compact footprint and high bandwidth, which has led to recent demonstrations at 112 and 128 Gb/s PAM4 [12], [13]. But MRMs are challenging to bias and introduce significant chirp. While EAMs require additional materials in the process, they also do show wavelength dependency and cannot be easily ported from C- to O-band. MZMs are typically larger and more power consuming but they operate in both $\mathrm{O}-$ and $\mathrm{C}$-band, are easy to bias, can be made without additional materials, have a higher linearity and are more suited for coherent communication. This makes them a popular choice as modulators.

Of the various physical mechanisms available to perform modulation in silicon MZMs, the plasma dispersion effect is most employed. It only requires doping the silicon waveguides. Three modes can be used to introduce phase shift: carrier injection, accumulation or depletion [14]. The latter is most often used because of the straightforward manufacturability and high-speed operation [15]. Note that by using other materials, higher modulation efficiencies and bandwidths may be obtained [8]-[10]. However, these approaches come at the cost of pure CMOS compatibility.

A myriad of innovative solutions have been demonstrated in recent years [1]-[7] to push the performance of silicon MZMs. Since the electro-optic effect in silicon is rather weak, long phase shifters are required [16]. Thus most high-speed designs incorporate traveling-wave structures to reach high bandwidths. The authors of [1] show that optimization of the doping levels and $\mathrm{PN}$-junction itself can be used to optimize the bandwidth- $\mathrm{V}_{\pi}$ trade-off, while [2] shows how termination mismatch of the traveling wave electrode can be used to enhance the electro-optic bandwidth. In [3] and [4], two PN-junctions are placed in series 
between two electrodes to half the load capacitance. This decreases the microwave attenuation and enhances the bandwidth. The advantage of loading a traveling-wave structure periodically with short phase-shifter segments instead of continuous loading is demonstrated in [5]. This technique leads to a so-called slowwave modulator which offers superior high-speed performance [5], [17]. A very interesting approach is taken in [7]. Instead of using a traveling-wave concept, the MZM is split into separate lumped segments that are driven separately. The authors of [7] drive the segments in such a way that a feed-forward equalizer (FFE) structure is obtained to improve the modulator bandwidth.

We proposed a novel slow-wave modulator design using carrier-depleted PN junctions in [18]. By careful analysis and design, a finite-impulse response (FIR) filter can be integrated in the structure. The frequency response of the FIR filter is applied onto the slow-wave modulator response allowing a wide range of FIR filter shapes. This allows to enhance the electro-optic bandwidth of the modulator or to introduce peaking to counteract bandwidth limitations in the driver, link or receiver. By adding FIR filters in the modulator, equalization elsewhere in the link can be minimized or even omitted. Making this technique very attractive for short-reach datacenter interconnects. The FIR filter can be integrated without introducing additional processing steps or by adjusting the electrical operation of the modulator. While the technique is demonstrated here on silicon photonics, it can be easily ported to other technology platforms.

In section II, we analyze the slow-wave modulator structure and explain how a FIR filter can be integrated in the structure. In section III-A and III-B, we cover the design and simulation of a standard and a shaped modulator. Electro-optic characterization of manufactured devices is covered in III-C. An optical link experiment is described in section IV. Compared to our previous work [18], we provide a full mathematical framework for the analysis of this novel design and discuss in-depth how this framework is used to design and simulate shaped slow-wave modulators.

\section{Slow-WaVE Modulator AS FIR FILTER}

We start from the standard slow-wave modulator design, as described in [17] and [19]. A differential drive push-pull MZM is chosen with series-push-pull (SPP) phase shifters to separate high-speed operation from the junction bias voltage. The electrodes are in a GSSG configuration as this provides advantages in drive voltage, inter-device crosstalk and driver integration [19], [20]. An example layout of such an MZM is given in Fig. 1.

\section{A. Standard Slow-Wave Modulator}

A very convenient way to analyze the electro-optic response of slow-wave modulators is provided by [17]. However, the ABCD-matrix approach only operates on 2-port structures, while a differential transmission line is modeled as a 4-port structure. In [19], it is shown that a differential MZM that is perfectly symmetrical and driven differentially can be fully described using its differential transmission line parameters.

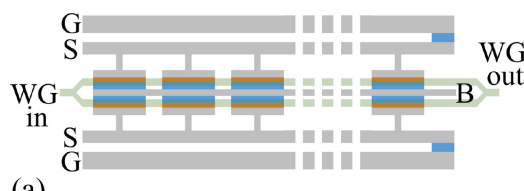

(a)

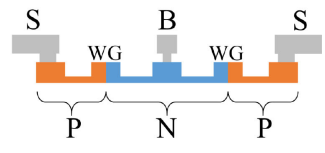

(b)

Fig. 1. (a) Layout of a differential drive push-pull MZM with SPP phase shifters, a GSSG electrode configuration and on-chip termination. The PNjunctions are biased using the B-line that does not carry any AC-signal. (b) Simplified cross section of a segment with PN-doped rib waveguides.

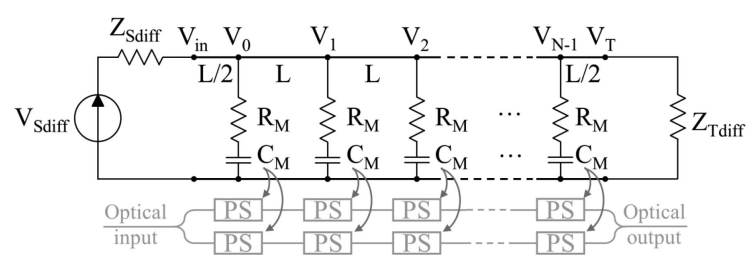

Fig. 2. Equivalent electrical and optical circuit of the slow-wave modulator in Fig. 1 with $\mathrm{N}$ segments. The electrical part only contains the differential mode parameters. The transmission line segments have a characteristic impedance $\mathrm{Z}_{\text {cdiff }}$ and propagation factor $\gamma_{\text {diff }}$. Both phase shifters are taken together in the equivalent $R C$ series network $R_{M} C_{M}$.

This simplifies the 4-port device to a 2-port device allowing the ABCD-matrix formalism to be applied. Since the proposed modulator structure from Fig. 1 is perfectly symmetrical and will be driven differentially, this technique can be applied. Converting the general MZM with $\mathrm{N}$ segments from Fig. 1 to its electrical equivalent leads to the circuit in Fig. 2.

The transmission line differential characteristic impedance and propagation factor are $\mathrm{Z}_{\text {cdiff }}$ and $\gamma_{\text {diff }}$. The pieces of transmission line between adjacent phase shifter segments have length L, while at the beginning and end of the first and last segment the length is $\mathrm{L} / 2$. The differential source impedance, source voltage and termination impedance are denoted $\mathrm{Z}_{\mathrm{Sdiff}}$, $\mathrm{V}_{\text {Sdiff }}$ and $\mathrm{Z}_{\text {Tdiff }}$. Each phase shifter is modeled as an $\mathrm{RC}$ series circuit $\mathrm{R}_{\mathrm{PN}} \mathrm{C}_{\mathrm{PN}}$. As they are placed in series, we find $\mathrm{R}_{\mathrm{M}}$ and $\mathrm{C}_{\mathrm{M}}$ equal to $2 \times \mathrm{R}_{\mathrm{PN}}$ and $\mathrm{C}_{\mathrm{PN}} / 2$ respectively. Note that it is also possible to include more elaborate equivalent circuits for the phase shifters.

As described in [17] and [19], we can now use the ABCDmatrix formalism to calculate the voltage over each $\mathrm{C}_{M}$. The ABCD matrix $T_{L}$ of the $L / 2$ piece of transmission line at the input is given by [21]:

$$
\left(\begin{array}{l}
V_{\text {in }} \\
I_{\text {in }}
\end{array}\right)=\underbrace{\left(\begin{array}{cc}
\cosh \left(\gamma_{\text {diff }} \frac{\mathrm{L}}{2}\right) & Z_{\text {cdiff }} \sinh \left(\gamma_{\text {diff }} \frac{\mathrm{L}}{2}\right) \\
Z_{\text {cdiff }}^{-1} \sinh \left(\gamma_{\text {diff }} \frac{\mathrm{L}}{2}\right) & \cosh \left(\gamma_{\text {diff } \left.\frac{\mathrm{L}}{2}\right)}^{2}\right.
\end{array}\right)}_{T_{\mathrm{L}}}\left(\begin{array}{c}
V_{0} \\
I_{0}
\end{array}\right)
$$

Since the $\mathrm{L} / 2$ piece of transmission line at the output has the same length, the ABCD matrix $T_{T}$ of that piece is equal to $T_{L}$ :

$$
\left(\begin{array}{c}
V_{\mathrm{N}-1} \\
I_{\mathrm{N}-1}
\end{array}\right)=T_{\mathrm{L}}\left(\begin{array}{c}
V_{\mathrm{\top}} \\
I_{\mathrm{\top}}
\end{array}\right)=T_{\mathrm{T}}\left(\begin{array}{c}
V_{\mathrm{T}} \\
I_{\mathrm{\top}}
\end{array}\right)
$$


Analogously, the $\mathrm{ABCD}$ matrix $\mathrm{T}_{\mathrm{C}}$ of the transmission lines segment between the phase shifters is given by:

$$
\left(\begin{array}{c}
V_{\mathrm{n}} \\
I_{\mathrm{n}}
\end{array}\right)=\underbrace{\left(\begin{array}{cc}
\cosh \left(\gamma_{\mathrm{diff}} L\right) & Z_{\mathrm{cdiff}} \sinh \left(\gamma_{\mathrm{diff}} L\right) \\
Z_{\mathrm{cdiff}}^{-1} \sinh \left(\gamma_{\mathrm{diff}} L\right) & \cosh \left(\gamma_{\mathrm{diff}} L\right)
\end{array}\right)}_{T_{\mathrm{C}}}\left(\begin{array}{c}
V_{\mathrm{n}+1} \\
I_{\mathrm{n}+1}
\end{array}\right)
$$

To complete, the $\mathrm{ABCD}$ matrix of the impedance of the phase shifters is given by [21]:

$$
T_{\mathrm{M}}=\left(\begin{array}{cc}
1 & 0 \\
\left(R_{\mathrm{M}}+\frac{1}{\mathrm{sC}_{M}}\right)^{-1} & 1
\end{array}\right)
$$

With $s=j \omega_{\mathrm{m}}$ and $\omega_{\mathrm{m}}$ the pulsation of the modulating signal. The voltage at the $\mathrm{n}^{\text {th }}$ segment can now be calculated by cascading the $\mathrm{ABCD}$ matrices of the elements preceding that specific segment, thus we find:

$$
\left(\begin{array}{c}
V_{\text {in }} \\
I_{\text {in }}
\end{array}\right)=T_{\mathrm{L}}\left(T_{\mathrm{M}} T_{\mathrm{C}}\right)^{\mathrm{n}}\left(\begin{array}{c}
V_{\mathrm{n}} \\
I_{\mathrm{n}}
\end{array}\right)
$$

With $\mathrm{n}$ from 0 to $\mathrm{N}-1$. Note that a matrix inversion is required to solve for $V_{n}$. Both the voltage and current at the input terminal of the modulator can be derived as follows:

$$
V_{\text {in }}=\frac{Z_{\text {in }}}{Z_{\text {Sdiff }}+Z_{\text {in }}} V_{\text {Sdiff }} \quad I_{\text {in }}=\frac{1}{Z_{\text {Sdiff }}+Z_{\text {in }}} V_{\text {Sdiff }}
$$

The input impedance $Z_{\text {in }}$ of the modulator can be derived from the $\mathrm{ABCD}$ matrix of the full modulator:

$$
Z_{\text {in }}=\frac{A_{\text {tot }} Z_{\text {Tdiff }}+B_{\text {tot }}}{C_{\text {tot }} Z_{\text {Tdiff }}+D_{\text {tot }}}
$$

With the ABCD matrix of the full modulator given by:

$$
\left(\begin{array}{ll}
A_{\text {tot }} & B_{\text {tot }} \\
C_{\text {tot }} & D_{\text {tot }}
\end{array}\right)=T_{\mathrm{L}}\left(T_{\mathrm{M}} T_{\mathrm{C}}\right)^{\mathrm{N}-1} T_{\mathrm{M}} T_{\mathrm{T}}
$$

Note that $\mathrm{V}_{\mathrm{n}}$ is the voltage over the $\mathrm{n}^{\text {th }}$ modulator segment. In the phase shifter, the voltage over $\mathrm{C}_{\mathrm{M}}$ gives rise to the optical phase shift. Thus the voltage divider $\mathrm{R}_{\mathrm{M}} \mathrm{C}_{\mathrm{M}}$ needs to be taken into account using the following transfer function:

$$
V_{\mathrm{n}}^{\prime}=\frac{1}{1+s R_{\mathrm{M}} C_{\mathrm{M}}} V_{\mathrm{n}}
$$

Each phase shifter introduces the phase shift at a different location on the modulator. To calculate the total introduced phase shift, a reference location on the MZM should be chosen at which all separate contributions can be combined. The most obvious location is the last segment. Thus the phase shift introduced in the $\mathrm{n}^{\text {th }}$ segment has to travel through N-1-n segments of physical length $\mathrm{L}$ with group index $\mathrm{n}_{\mathrm{o}}$. Since the optical waveguides run parallel along the electrodes, the physical distance between adjacent segments in the electrical and optical domain is both $\mathrm{L}$. This results in the following delay correction factor:

$$
V_{\mathrm{n}}^{\prime \prime}=V_{\mathrm{n}}^{\prime} \exp \left(-j \beta_{\mathrm{o}}(N-1-n) L\right)
$$

With the propagation factor $\beta_{\mathrm{o}}$ given by:

$$
\beta_{\mathrm{o}}=\frac{\omega_{\mathrm{m}} n_{\mathrm{O}}}{c}
$$

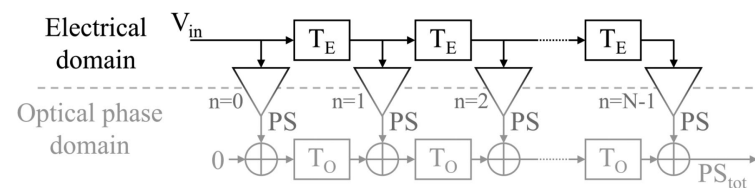

Fig. 3. Simplified block diagram of a single arm in a slow-wave modulator.

The resulting voltage that introduces phase shift is then:

$$
V_{\text {tot }}=\frac{1}{N} \sum_{\mathrm{n}=0}^{\mathrm{N}-1} \frac{\exp \left(-j \beta_{\mathrm{o}}(N-1-n) L\right)}{1+s R_{\mathrm{M}} C_{\mathrm{M}}} V_{\mathrm{n}}
$$

Which is the same as found in [17]. The phase shift (in radians) between both arms at the MZM output is now given by:

$$
P S_{\mathrm{tot}}=\pi \frac{V_{\mathrm{tot}}}{V_{\pi}}
$$

With $\mathrm{V}_{\pi}$ calculated over the full MZM, not a single segment. This total phase shift gives rise to the intensity modulation at the output of the MZM. Using this analysis, the small-signal electro-optic response of a slow-wave modulator can be derived. Moreover, using some minor adjustments, a FIR filter structure can be identified.

\section{B. FIR Filtering in Slow-Wave Modulators}

The equivalent electrical schematic from Fig. 2 can be further abstracted. To be able to calculate the full phase shift introduced by the segments, an additional delay correction was introduced at the optical side. This delay is linked to the distance in the optical domain between two adjacent segments. In the electrical domain, a similar delay is present due to the transmission lines. This delay is already accounted for in the ABCD matrices. If we draw a block diagram of the modulator that only includes these electrical and optical domain delays and the phase shifters, we obtain Fig. 3. This diagram only describes the behavior of one arm of the MZM, the other arm is identical, but is driven by opposite signals such that opposite phase shifts are introduced. Some simplifications were used for this description: the transmission line segments are replaced by fixed delays (no attenuation, perfectly matched to the source impedance), the transmission lines are perfectly terminated (no reflected waves) and each phase shifter introduces a frequency-independent phase shift PS at its output. No simplifications are carried out at the optical side: the optical delays are the same as those derived in the previous section.

From this block diagram, the equivalency to a FIR filter becomes apparent [22]. In a typical modulator design, the velocity of the electrical and optical wave are matched such that they simultaneously arrive at subsequent segments. In that case, $T_{E}$ is equal to $\mathrm{T}_{\mathrm{O}}$ and the full phase shift is the sum of all individual phase shifts. If no velocity match is achieved, a delay factor $\mathrm{T}_{\mathrm{O}}-\mathrm{T}_{\mathrm{E}}$ is present between subsequent segments. Thus the total phase shift is given by:

$$
\mathrm{PS}_{\text {tot }}=\sum_{\mathrm{n}=0}^{\mathrm{N}-1} \exp \left(-\mathrm{j}(\mathrm{N}-1-\mathrm{n})\left(\mathrm{T}_{\mathrm{O}}-\mathrm{T}_{\mathrm{E}}\right) \omega_{\mathrm{m}}\right) \cdot \mathrm{PS}
$$




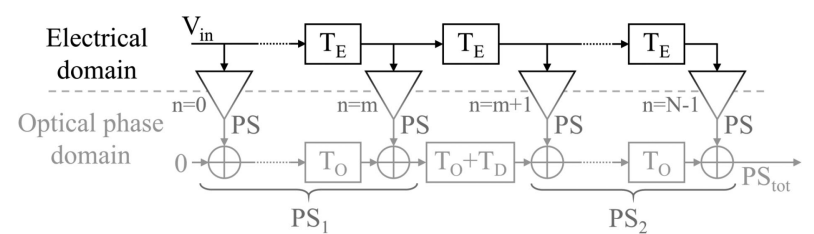

Fig. 4. Simplified block diagram of a velocity matched modulator $\left(\mathrm{T}_{\mathrm{E}}=\mathrm{T}_{\mathrm{O}}\right)$ where an additional optical delay line is incorporated between the $\mathrm{m}^{\text {th }}$ and $\mathrm{m}+1^{\text {th }}$ segments.

This transfer function consists of a sum of delays yielding the well-known low-pass filtering due to velocity mismatch [6]. In order to add fully controllable FIR filters, we need to create 'taps' with a specific weight and delay [22]. Starting from a velocity matched modulator, one can add an optical delay line with delay $T_{D}$ between the $m^{\text {th }}$ and $m+1^{\text {th }}$ phase shifter. This yields the equivalent block diagram of Fig. 4. Note that adding delay in the optical domain is preferred: the optical waveguides have a smaller bend radius, lower losses and much higher bandwidth than the transmission line electrodes.

As the modulator is velocity matched, all phase shifts introduced before the delay $T_{D}$ can be added together to obtain a total phase shift $\mathrm{PS}_{1}$ of $(\mathrm{m}+1) \times \mathrm{PS}$. The same can be done with all phase shifts introduced after the delay, resulting in a total phase shift $\mathrm{PS}_{2}$ equal to $(\mathrm{N}-\mathrm{m}-1) \times \mathrm{PS}$. At the transition between the $m^{\text {th }}$ and $(m+1)^{\text {th }}$ segments, a net delay of $T_{O}+T_{D}-T_{E}$ is introduced. Since $T_{E}=T_{O}$, this simplifies to $T_{D}$. When observed at the output, $\mathrm{PS}_{2}$ directly contributes to $\mathrm{PS}_{\text {tot }}$ while $\mathrm{PS}_{1}$ experiences an additional delay of $\mathrm{T}_{\mathrm{D}}$, this results in the following total phase shift:

$$
\mathrm{PS}_{\text {tot }}=(\mathrm{N}-\mathrm{m}-1) \mathrm{PS}+(\mathrm{m}+1) \mathrm{PS} \cdot \exp \left(-\mathrm{j} \mathrm{T}_{\mathrm{D}} \omega_{\mathrm{m}}\right)
$$

This corresponds to a z-domain representation of a FIR filter by substituting $z=\exp \left(j T_{\mathrm{D}} \omega_{\mathrm{m}}\right)$ :

$$
\mathrm{PS}_{\text {tot }}=(\mathrm{N}-\mathrm{m}-1) \mathrm{PS}+(\mathrm{m}+1) \mathrm{PS} \cdot \mathrm{z}^{-1}
$$

By tuning the position $m$ of the optical delay line, the relative tap coefficients can be set. Tuning the length of the optical delay line changes $T_{D}$. Note that multiple optical delay lines can be added to create higher order transfer functions. The optical delay line should be inserted in both arms of the MZM.

However, only positive coefficients can be made this way. By using negative coefficients in FIR filters, peaking in the frequency response can be introduced. Note that the proposed block diagrams only describe the behavior of one arm. As the modulator is symmetrical and driven differentially, the other arm introduces exactly the opposite phase shift (optical delay lines should be introduced in both arms). By crossing the optical waveguides of both arms while keeping the electrodes unaltered, negative coefficients can be achieved. This is demonstrated in the block diagram of Fig. 5 where a crossing between the arms and a delay line in each arm are inserted between the $\mathrm{m}^{\text {th }}$ and $(\mathrm{m}+1)^{\mathrm{th}}$ segments. Low-loss, low-cross-talks crossings are available in silicon photonics [23]. We now determine the phase shift $\mathrm{PS}_{\text {tot }}$ at the output of the top arm. All phase shifts introduced after the crossing can still be added together to obtain

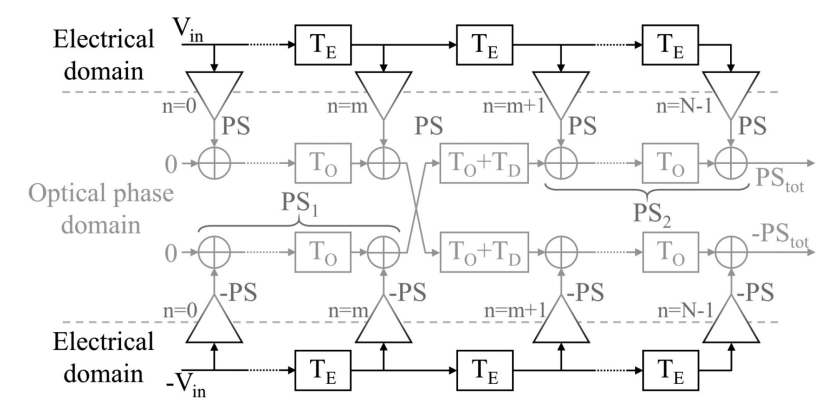

Fig. 5. Simplified block diagram of both arms of a velocity matched modulator $\left(\mathrm{T}_{\mathrm{E}}=\mathrm{T}_{\mathrm{O}}\right)$ where an additional crossing and delay line are incorporated between the $\mathrm{m}^{\text {th }}$ and $\mathrm{m}+1^{\text {th }}$ segments.

a total phase shift $\mathrm{PS}_{2}$ of $(\mathrm{N}-\mathrm{m}-1) \times \mathrm{PS}$. Before the crossing, the individual phase shifts can also be added, but because of the crossing, the sign is inverted. This yields a negative phase shift $\mathrm{PS}_{1}$ for the top arm of $-(\mathrm{m}+1) \times \mathrm{PS}$. So we obtain:

$$
\mathrm{PS}_{\text {tot }}=(\mathrm{N}-\mathrm{m}-1) \mathrm{PS}-(\mathrm{m}+1) \mathrm{PS} \cdot \mathrm{z}^{-1}
$$

The crossing and delay lines introduce additional delays. This had to be adjusted to obtain the same delay as when only a delay line without crossing is inserted between two segments. Similar to the delay-line-only case, the position of the crossing determines the tap weights while the length of the delay line determines $T_{D}$. Furthermore, the delay from Fig. 4 and crossing from Fig. 5 can be used on different locations to obtain higherorder transfer functions with both positive and negative taps. The maximal order transfer function is limited to the number of segments minus one, the sum of the absolute values of the tapweights will always be equal to the total number of segments and the smallest value in which a tap can be adjusted is one over the number of taps.

As outlined at the beginning of this subsection, several simplifications were used in this block diagram approach. We can now adjust Eq. (12) from subsection II-A to incorporate the FIR filtering without the simplifications. For each segment, an additional phase factor is required to account for the added optical delay between that segment and the last segment, as the reference position to combine all phase shifts is this last segment. An additional sign is required to account for the arm in which the phase shift was introduced. We obtain the following adjusted equation:

$$
V_{\text {tot }}=\frac{1}{\mathrm{~N}} \sum_{\mathrm{n}=0}^{\mathrm{N}-1} \frac{\exp \left(-\mathrm{j} \beta_{o}(\mathrm{~N}-1-\mathrm{n}) \mathrm{L}-\mathrm{j} \mathrm{T}_{d} \mathrm{D}(\mathrm{n})\right)}{1+\mathrm{SR}_{M} \mathrm{C}_{M}}(-1)^{\mathrm{C}(\mathrm{n})} V_{\mathrm{n}}
$$

with $\mathrm{D}(\mathrm{n})$ and $\mathrm{C}(\mathrm{n})$ the number of delay lines and crossing that follow on the $\mathrm{n}^{\text {th }}$ segment. Note that each crossing also includes a delay line. We assume all delay lines to have the same delay $\mathrm{T}_{\mathrm{D}}$. The total induced phase shift (in radians) between both arms can still be calculated using Eq. (13).

\section{Design of A ShaPed Slow-WAVE Modulator}

A slow-wave modulator operating in the C-band is designed on imec's iSiPP50G technology platform [24] to validate the concept, the electro-optic modeling and the design procedure. 

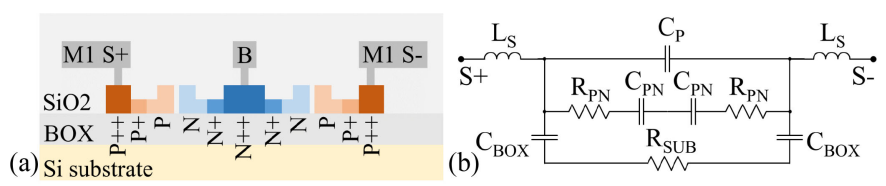

Fig. 6. (a) Cross section of the simulated segment with the SPP phase shifters. (b) Equivalent electrical schematic for the cross-section in (a).

The electrical characteristics of both the transmission line pieces and the phase shifters have been simulated using ADS Momentum. Those simulation results are combined afterwards to obtain the electro-optic response of the slow-wave modulator. First, we will briefly discuss the design of the standard modulator, then we will apply and analyze the FIR filter technique.

\section{A. Standard Slow-Wave Modulator Design}

Using ADS Momentum, a piece of differential transmission line was simulated. The S-parameters from this simulation are exported to extract the differential characteristic impedance and differential propagation factor. From this, the ABCD matrices of all transmission line segments can be derived for an arbitrary length. The length of the electrodes can be easily adjusted afterwards without having to resimulate, the other dimensions are fixed when simulating in ADS. To incorporate the phase shifter with the cross section given in Fig. 6(a), the impedance can be measured and fitted on the equivalent electrical circuit of Fig. 6(b). However, as no measurement was available in our case, a phase shifter with the cross section of Fig. 6(a) was simulated using ADS Momentum. The doped layers are modeled as conductors, the conductivity is provided in the platform documentation. As the simulator cannot fully model a carrier-depleted PN-junction, the depletion region is modeled as a gap between the $\mathrm{P}$ and $\mathrm{N}$ doped layers which is filled with silicon dioxide. The width of the gap is adjusted to obtain the correct junction capacitance for a certain reverse bias voltage. This junction capacitance is also provided in the platform documentation. The gap behaves like a parallel plate capacitor, as the width (40-60 $\mathrm{nm}$ for a reverse bias between 0 and $2 \mathrm{~V}$ ) is typically much lower than the height of the doped layers $(220 \mathrm{~nm})$. The dimensions of the doped layers are identical to the default phase shifter as provided in the process design kit (PDK). The impedance found in the ADS simulation is fitted onto the equivalent electrical network from Fig. $6(\mathrm{~b})$. Where $\mathrm{L}_{\mathrm{s}}$ is the contacting inductance, $\mathrm{C}_{\mathrm{P}}$ resembles parallel capacitance over the phase shifter, $R_{P N}$ and $C_{P N}$ resemble the resistance towards the $\mathrm{PN}$-junction and the actual junction capacitance, $\mathrm{C}_{\mathrm{BOX}}$ the buried oxide capacitance and $\mathrm{R}_{\mathrm{SUB}}$ the substrate resistance. This equivalent network is combined with the transmission line parameters to calculate the full slow-wave modulator response. Note that Eq. (4) and Eq. (9) should be adjusted to accommodate the network of Fig. 6(b).

The phase shifter with the cross section of Fig. 6 is simulated with a length of 100, 150 and $200 \mu \mathrm{m}$ in ADS. Each time a fit with the equivalent network has been made. For a fixed gap width (i.e. fixed reverse bias voltage), $\mathrm{C}_{\mathrm{P}}, \mathrm{R}_{\mathrm{PN}}, \mathrm{C}_{\mathrm{PN}}, \mathrm{C}_{\mathrm{BOX}}, \mathrm{R}_{\mathrm{SUB}}$ were proportional to the segment length and $\mathrm{L}_{\mathrm{s}}$ was independent

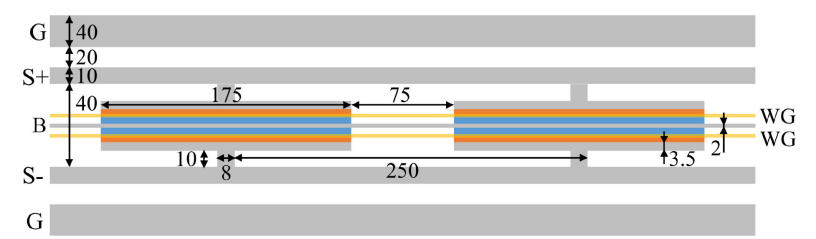

Fig. 7. Dimensions of segments and traveling-wave electrodes of the modulator. All dimensions are in $\mu \mathrm{m}$.

Fig. 8. Differential characteristic impedance, microwave attenuation and phase index of the slow-wave modulator with the electrode layout of Fig. 7.

on segment length. This allows to optimize the segment length based on the developed equivalent network without having to resimulate the segment in ADS.

The unloaded transmission lines and segments were combined and transmission line dimensions and segment length were optimized to obtain a high-bandwidth modulator with a matched input: a differential characteristic impedance of $100 \Omega$, minimal attenuation and a phase index close to 4.26 , to velocity match the electrical and optical wave. The group index of the C-band optical waveguide is 4.26. Furthermore, there should be sufficient place between two subsequent segments to insert the delay and crossing. In our case, this is at least $75 \mu \mathrm{m}$. The optimal dimensions for both the traveling-wave electrodes and the phase shifter segments are shown in Fig. 7. The PN-junctions are simulated for a reverse bias voltage of 0 and $2 \mathrm{~V}$. For the optimization, we assume a reverse bias of $2 \mathrm{~V}$.

The phase shifter segments are $175 \mu \mathrm{m}$ long and have a pitch of $250 \mu \mathrm{m}$, this provides a tradeoff between modulation efficiency, characteristic impedance, attenuation and refractive index. Shorter segments result in a higher characteristic impedance and lower attenuation, but also a worse modulation efficiency. The simulated characteristic impedance of the loaded transmission line is around $75 \Omega$, as shown in Fig. 8. While this is lower than the intended $100 \Omega$ differential impedance, when properly terminated this will still yield a low reflection coefficient. The phase index of the electrical signal is close to the group index of the optical signal, being 4.26.

For the standard slow-wave modulator design, we chose a modulator with 10 segments. Thus the total modulator length is $2.5 \mathrm{~mm}$ of which $1.75 \mathrm{~mm}$ effective phase shifters. The $\mathrm{V}_{\pi} \mathrm{L}_{\pi}$ of the phase shifters is $24.6 \mathrm{~V} . \mathrm{mm}$ at a reverse bias of $2 \mathrm{~V}$ at $1550 \mathrm{~nm}$, resulting in a $\mathrm{V}_{\pi}$ of $14 \mathrm{~V}$. The optical attenuation of the phase shifters is $1.5 \mathrm{~dB} / \mathrm{mm}$, thus a total insertion loss of $2.6 \mathrm{~dB}$ is expected. Given the characteristic impedance of $75 \Omega$, a differential termination impedance of $70 \Omega$ is chosen. The simulated electro-optic response of this configuration is given in Fig. 10 ("Standard" curve). The $3 \mathrm{~dB}$ bandwidth is $30.6 \mathrm{GHz}$ 

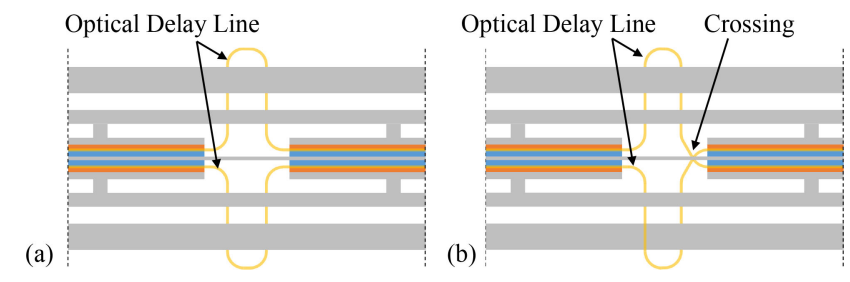

Fig. 9. (a) Implementation of a delay line between two segments, (b) implementation of a delay line and crossing between to segments.

Fig. 10. (a) Simulated electro-optic response for the standard modulator, for a crossing between the $1^{\text {st }}-2^{\text {nd }}, 2^{\text {nd }}-3^{\text {rd }}$ and $3^{\text {rd }}-4^{\text {th }}$ segments (delay line length $500 \mu \mathrm{m}$ ). (b) Normalized Electro-optic response of the modulator with crossing between the $2^{\text {nd }}-3^{\text {rd }}$ segments for various lengths of delay line. All simulations assume a reverse bias of $2 \mathrm{~V}$.

and the simulated input reflection coefficient (for a $100 \Omega$ source impedance) is below $-15 \mathrm{~dB}$ up to $50 \mathrm{GHz}$.

\section{B. Embedding FIR Filtering}

As explained in section II-B, by adding optical delay lines and crossings between subsequent phase shifting segments, a FIR filter can be created. An implementation of how such a delay line and crossing can be implemented is shown in Fig. 9. The delay lines are implemented using the PDK strip waveguides. The C-band waveguides have a low loss of $0.14 \mathrm{~dB} / \mathrm{mm}$. The PDK crossings feature $0.3 \mathrm{~dB}$ insertion loss and $-30 \mathrm{~dB}$ crosstalk. Remark that no other changes are required, thus the electrical part of the modulator is untouched.

In order to demonstrate the effect of the FIR filter, the slowwave modulator designed in the previous section is adapted. The simulated electro-optic response for the standard modulator and modulator with the crossing on various positions is given in Fig. 10(a). In all three cases, the delay line length is $500 \mu \mathrm{m}$, which is equivalent to $7 \mathrm{ps}$. Using the block-diagram approach of section II-B, we can derive the transfer function for the various crossing positions. When the crossing is between the $1^{\text {st }}-2^{\text {nd }}, 2^{\text {nd }}-3^{\text {rd }}$ and $3^{\text {rd }}-4^{\text {th }}$ segments, the transfer functions are respectively $9 \mathrm{PS}-\mathrm{PS} \cdot \mathrm{z}^{-1}, 8 \mathrm{PS}-2 \mathrm{PS} \cdot \mathrm{z}^{-1}$, $7 \mathrm{PS}-3 \mathrm{PS} \cdot \mathrm{z}^{-1}$. Thus placing the crossing further down the modulator increases the negative tap in the FIR filter, which results in more peaking but also a lower DC-response. This is confirmed in the simulation of Fig. 10(a). By adjusting the delay line length, the peaking frequency can be shifted. This is demonstrated in Fig. 10(b). The crossing is placed between the $2^{\text {nd }}-3^{\text {rd }}$ segments and the delay length is swept from $250 \mu \mathrm{m}$ to $1000 \mu \mathrm{m}$. All electro-optic responses in Fig. 10(b) have been normalized at $1 \mathrm{GHz}$ to ease comparison. By increasing the delay line length, the peaking frequency drops as expected. By
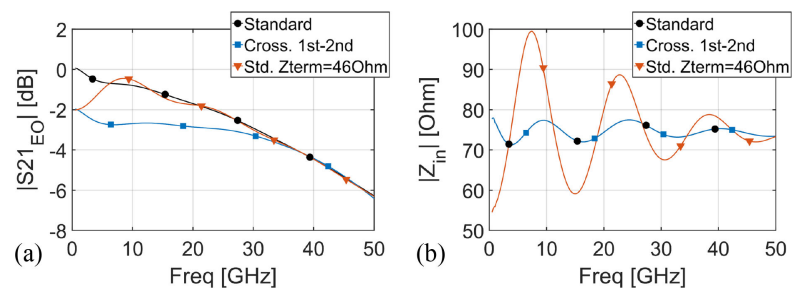

Fig. 11. (a) Electro-optic response for the standard modulator, modulator with a crossing between the $1^{\text {st }}-2^{\text {nd }}$ segments and standard modulator with lower termination(b) Input impedances of the modulators used in (a). All simulations assume a reverse bias of $2 \mathrm{~V}$.

adjusting the crossing position and the delay line length, both the amount of peaking and the peaking frequency can be controlled.

A popular way to enhance the bandwidth of traveling wave modulators is by lowering the termination impedance [2]. To compare with our technique, we have simulated the standard modulator and the modulator with a crossing between the $1^{\text {st }}$ $2^{\text {nd }}$ segment. Both the FIR filter technique as well as lowering the termination impedance impose a penalty on the modulation efficiency. To compare, we have lowered the termination impedance of the standard modulator from $70 \Omega$ to $46 \Omega$ in order to obtain the same penalty as placing a crossing between the $1^{\text {st }}-2^{\text {nd }}$ segment. The resulting electro-optic responses are shown in Fig. 11(a). With the crossing and optimized delay line $(750 \mu \mathrm{m})$, the electro-optic bandwidth is $43.7 \mathrm{GHz}$, compared to $42.9 \mathrm{GHz}$ with the lower termination impedance. Both cases provide a significant improvement compared to the original standard modulator $(30.6 \mathrm{GHz})$. However, the response obtained by lowering the termination impedance shows $1.5 \mathrm{~dB}$ peaking, while the response obtained by FIR filtering is much smoother. Moreover, by adjusting the termination impedance, the input impedance of the modulator shows significant variation (>25\%), see Fig. 11(b). This complicates integration with a driver while also affecting the driver performance. If even higher bandwidths are required, the FIR filter technique can be easily adjusted while lowering the termination impedance will lead to excessive peaking and variations in input impedance.

In our technique, the accuracy of the tap weights and number of taps are limited by the number of segments. Furthermore, the filter is fixed in the layout. Electrical feed-forward equalizers can provide high accuracy tap weights, scale better to more taps and are reconfigurable. However, as the FIR filters are integrated in the modulators, they can take over the electrical equalization partially or completely in certain cases to provide a power and area-efficient way of equalization.

\section{Final Designs and Electro-Optic Characterization}

Two designs have been manufactured in imec's iSiPP50G platform [24], both are shown in Fig. 12(a). The standard design and a shaped design which includes a crossing between the $3^{\text {rd }}-4^{\text {th }}$ segments and a delay between the $4^{\text {th }}-5^{\text {th }}$ segments. The realized transfer function is $6 \mathrm{PS}+\mathrm{PS} \cdot \mathrm{z}^{-1}-3 \mathrm{PS} \cdot \mathrm{z}^{-2}$, while the delay line length is $500 \mu \mathrm{m}(7 \mathrm{ps})$. The simulated electro-optic responses are shown in Fig. 12(b). The $3 \mathrm{~dB}$ bandwidth of the standard modulator is $30.6 \mathrm{GHz}$. The shaped modulator introduces $4.8 \mathrm{~dB}$ peaking at $25.7 \mathrm{GHz}$. 

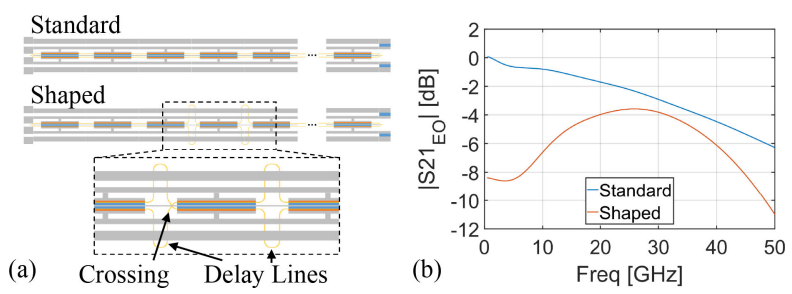

Fig. 12. (a) Layout of the fabricated designs (b) Simulated electro-optic response of the designs in (a) for a reverse bias of $2 \mathrm{~V}$.

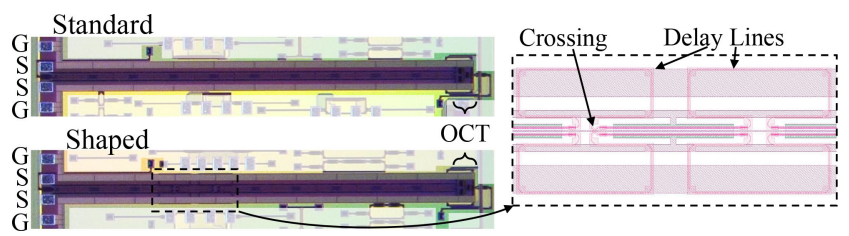

Fig. 13. Micrograph of the layout of both modulators with an inset of the crossing and delay.
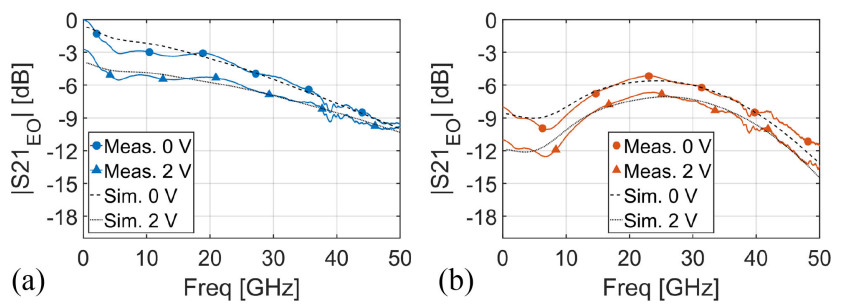

Fig. 14. Measured and simulated electro-optic response of the standard modulator (a) and the shaped modulator (b). The EO-response of the standard modulator is normalized to $0 \mathrm{~dB}$ at $\mathrm{DC}$, all other EO responses are referenced to this response.

The manufactured devices are shown in Fig. 13, both feature the GSSG driving scheme, the separate biasing line for the PNjunctions, the $70 \Omega$ on-chip termination (OCT) and thermo-optic heaters to bias the modulator at the quadrature point. The insets show the details of the crossing and delay lines. As these were partially hidden below the metal, a view of the designed layout is given.

The measured $V_{\pi}$ at a reverse bias of 1 and $2 \mathrm{~V}$ is respectively 11.8 and $14.0 \mathrm{~V}$ for the standard modulator, while being 29.6 and $35.1 \mathrm{~V}$ respectively for the shaped design. From the FIR transfer function, it can be observed that only 4 segments are actually contributing in DC instead of 10 . This results in a $V_{\pi}$ that is 2.5 times higher. Note that if a lower negative tapweight is used, the $\mathrm{V}_{\pi}$ penalty will be lower. The insertion loss at a reverse bias of 1 and $2 \mathrm{~V}$ is respectively 2.6 and $2.5 \mathrm{~dB}$ for the standard modulator while being 3.1 and $3.0 \mathrm{~dB}$ respectively for the shaped modulator. The additional insertion loss of the shaped modulator is attributed to the delay lines and the crossings.

The measured electro-optic response of the standard and shaped modulator at a reverse bias of 0 and $2 \mathrm{~V}$ is given in Fig. 14 . The agreement between the simulations and measurements is good. Only for the standard modulator at lower frequencies, there is some deviation. We expect that this is caused by the termination impedance that is slightly higher than expected, causing this additional ripple and roll-off in the low-GHz region. Increasing the termination impedance from $70 \Omega$ to $90 \Omega$ in

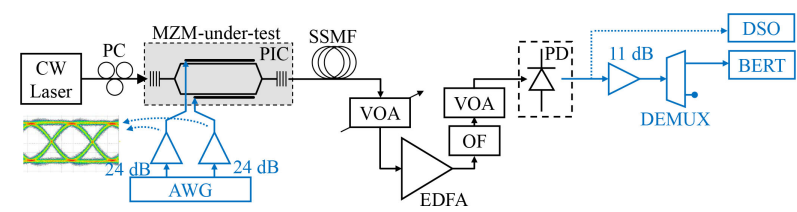

Fig. 15. Experiment setup for the transmission experiment. CW: continuouswave laser, PC: polarization controller, AWG: arbitrary waveform generator, VOA: variable optical attenuator, OF: optical filter, DSO: digital sampling oscilloscope, BERT: bit error rate-tester
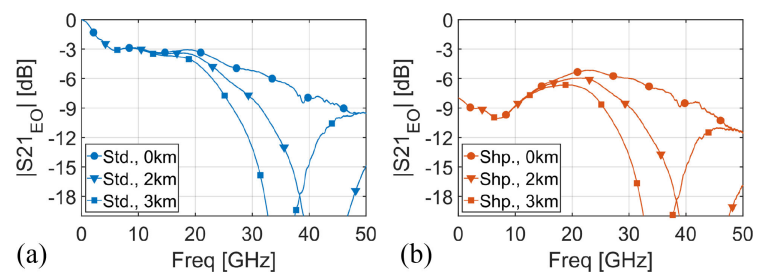

Fig. 16. Cascade of the measured electro-optic response of the modulator and the simulated fiber channel ( 0,2 and $3 \mathrm{~km}$ fiber) for the standard modulator (a) and the shaped modulator (b). The EO-response of the standard modulator is normalized to $0 \mathrm{~dB}$ at $\mathrm{DC}$, all other $\mathrm{EO}$ responses are referenced to this response. The modulators were biased at $0 \mathrm{~V}$.

simulation results in a much better fit. The bandwidth of the standard modulator is $21 \mathrm{GHz}$ at $0 \mathrm{~V}$ and $25.1 \mathrm{GHz}$ at $2 \mathrm{~V}$ reverse bias. The shaped modulator has $3.2 \mathrm{~dB}$ peaking at 23.2 $\mathrm{GHz}$ at $0 \mathrm{~V}$ reverse bias and $4.8 \mathrm{~dB}$ peaking at $23.8 \mathrm{GHz}$ at $2 \mathrm{~V}$ reverse bias.

\section{TRANSMISSION EXPERIMENT}

To finalize, we briefly go through a C-band transmission experiment using $56 \mathrm{~Gb} / \mathrm{s}$ non-return-to-zero (NRZ) data (PRBS $\left.2^{15}-1\right)$ and compare the performance of the standard and shaped design. Due to the bandwidth limitation imposed by chromatic dispersion, it is expected that the shaped design will have a lower bit-error-rate (BER) in the dispersion limited case. The experiment setup is shown in Fig. 15.

A laser is set to emit $13 \mathrm{dBm}$ of power at $1550 \mathrm{~nm}$. The light is sent into the PIC through a polarization sensitive grating coupler, a polarization controller is used to minimize the insertion loss. The AWG generates a differential NRZ signal, the amplifiers boost this to $4 \mathrm{~V}_{\mathrm{pp} \text {,diff }}$ for a $100 \Omega$ differential load. The simulated input impedance of our MZM is around $70 \Omega$, thus the voltage at the input of the modulator will be around $3.3 \mathrm{~V}_{\mathrm{pp} \text {,diff. }}$. The average power launched into the fiber is around $1 \mathrm{dBm}$. The $12 \mathrm{~dB}$ insertion loss is caused by the grating couplers $(2 \times 3 \mathrm{~dB})$, the modulator biased at quadrature $(3 \mathrm{~dB})$ and the insertion loss of the phase shifters $(3 \mathrm{~dB})$. Fiber reaches of 0,2 and $3 \mathrm{~km}$ were used. A VOA before the EDFA is used to control the input power. The EDFA, optical filter $(1.2 \mathrm{~nm} \mathrm{BW}), \mathrm{VOA}$ and $70 \mathrm{GHz}$ photodiode (PD) act as a reference receiver. The signal is sent to a DSO to capture the eye diagrams, or demuxed into $2 \times 28 \mathrm{~Gb} / \mathrm{s}$ of which one stream is used for BER analysis. The $11 \mathrm{~dB}$ linear, broadband amplifier with $67 \mathrm{GHz}$ bandwidth is used to amplify the signal before demuxing.

In Fig. 16, we have cascaded the measured electro-optic response of the modulators with the analytical transfer function 


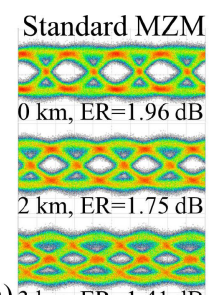

(a) $3 \mathrm{~km}, \mathrm{ER}=1.41 \mathrm{~dB}$

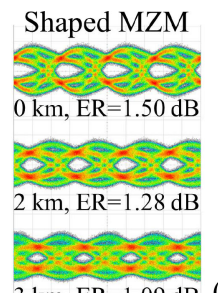

$3 \mathrm{~km}, \mathrm{ER}=1.09 \mathrm{~dB}(\mathrm{~b})$

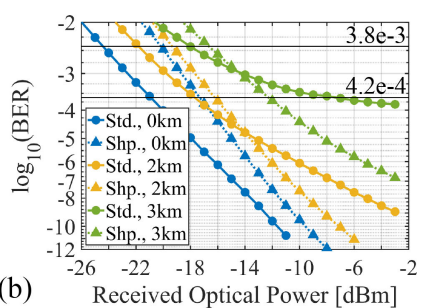

Received Optical Power [dBm]

Fig. 17. (a) Measured eye diagrams at $56 \mathrm{~Gb} / \mathrm{s} \mathrm{NRZ,} \mathrm{(b)} \mathrm{corresponding} \mathrm{BER}$ measurements as a function of average receive power.

of a chromatic dispersive channel from [25]. The dispersion parameter used for the simulation was $17 \mathrm{ps} /(\mathrm{nm} . \mathrm{km})$ at $1550 \mathrm{~nm}$, fiber loss was omitted to ease the comparison. At the Nyquist frequency of the $56 \mathrm{~Gb} / \mathrm{s}$ signal, thus $28 \mathrm{GHz}$, the response of the standard modulator cascaded with $3 \mathrm{~km}$ fiber has dropped $10 \mathrm{~dB}$ with respect to the $\mathrm{DC}$ value. While the response of the shaped modulator and $3 \mathrm{~km}$ fiber only dropped by $3 \mathrm{~dB}$ with respect to its corresponding $\mathrm{DC}$ value, thanks to the additional peaking.

The measured eye diagrams and BER measurements for the standard and shaped modulator over 0,2 and $3 \mathrm{~km}$ fiber are shown in Fig. 17(a) and (b). The average optical receive power is measured at the input of the EDFA. The reverse bias was $0.5 \mathrm{~V}$ to optimize the extinction ratio. As could be expected, the eyes of the shaped modulator are smaller due to the lower modulation efficiency, but they do show more peaking resulting in a cleaner eye after $3 \mathrm{~km}$ fiber. This is also observed in the BER measurements: at low optical receive powers, the link is noise limited such that the standard modulator has a better BER. The power penalty between the standard and shaped modulator without fiber $(0 \mathrm{~km})$ is slightly higher than $4 \mathrm{~dB}$ at a BER of $3.8 \times$ $10^{-3}$. At these low powers, the BER is dominated by the SNR and not by ISI. As the noise is identical in both cases, this $4 \mathrm{~dB}$ difference can be traced back to the $4 \mathrm{~dB}$ (factor 2.5) difference in $V_{\pi}$ between the standard and shaped modulator. At higher optical receive powers and longer fiber reaches ( 2 and $3 \mathrm{~km}$ ), the peaking of the shaped modulator results in a significantly better BER.

\section{CONCLUSION}

In this paper, we described how FIR filters can be embedded in slow-wave Mach-Zehnder modulators by adding optical delay lines and optical crossings between phase shifter segments. The technique proposed in [18] was further elaborated and analyzed in this work. The proposed technique does not require any changes to the electrical part of the modulator, nor does it require additional processing. Simulation models were devised to analyze and design shaped slow-wave MZM designs. Various cases were considered and compared to show how the response of the modulator could be optimized. A standard and shaped MZM were designed in imec's iSiPP50G silicon photonic platform to verify the modeling approach. Finally, a C-band transmission experiment with $56 \mathrm{~Gb} / \mathrm{s} \mathrm{NRZ} \mathrm{data} \mathrm{over} 3 \mathrm{~km}$ fiber was conducted to compare a standard and shaped modulator. The shaped modulator was designed to introduce peaking in order to counteract the bandwidth limitation of the fiber chromatic

dispersion. Eye diagrams and BER measurements show the advantage of adding shaping in the MZM.

\section{REFERENCES}

[1] D. Petousi et al., "Analysis of optical and electrical tradeoffs of travelingwave depletion-type Si Mach-Zehnder modulators for high-speed operation," IEEE J. Sel. Top. Quantum Electron., vol. 21, no. 4, pp. 199-206, 2015.

[2] H. Yu and W. Bogaerts, "An equivalent circuit model of the traveling wave electrode for carrier-depletion-based silicon optical modulators," $J$. Lightw. Technol., vol. 30, no. 11, pp. 1602-1609, Jun. 2012.

[3] P. Dong, L. Chen, and Y. kai Chen, "High-speed low-voltage single-drive push-pull silicon Mach-Zehnder modulators," Opt. Express, vol. 20, no. 6, pp. 6163-6169, Mar. 2012.

[4] A. Samani et al., "A low-voltage 35-GHz silicon photonic modulatorenabled 112-Gb/s transmission system," IEEE Photon. J., vol. 7, no. 3, pp. 1-13, 2015.

[5] D. Patel et al., "Design, analysis, and transmission system performance of a $41 \mathrm{GHz}$ silicon photonic modulator," Opt. Express, vol. 23, no. 11, pp. 14 263-14 287, Jun. 2015.

[6] R. Ding et al., "High-speed silicon modulator with slow-wave electrodes and fully independent differential drive," J. Lightw. Technol., vol. 32, no. 12, pp. 2240-2247, 2014.

[7] B. G. Lee et al., "SiGe-driven hybrid-integrated silicon photonic link using optical-domain equalization," J. Lightw. Technol., vol. 37, no. 1, pp. 89-94, 2019.

[8] M. He et al., "High-performance hybrid silicon and lithium niobate MachZehnder modulators for $100 \mathrm{Gbps}$ and beyond," Nat. Photon., vol. 13, no. 5, pp. 359-364, 2019.

[9] H. Zwickel et al., "Silicon-organic hybrid ( $\mathrm{SOH})$ modulators for intensitymodulation / direct-detection links with line rates of up to $120 \mathrm{Gbit} / \mathrm{s}$," Opt. Express, vol. 25, no. 20, pp. 23 784-23 800, Oct. 2017.

[10] Y. Ogiso et al., "Over $67 \mathrm{GHz}$ bandwidth and $1.5 \mathrm{~V} \mathrm{~V} \pi$ InP-based optical IQ modulator with n-i-p-n heterostructure," J. Lightw. Technol., vol. 35, no. 8, pp. 1450-1455, 2017.

[11] E. El-Fiky et al., "112 Gb/s PAM4 transmission over 2 km SMF using a C-band GeSi electro-absorption modulator," in Proc. Opt. Fiber Comm. Conf. Expo., 2018, p. W2A.16.

[12] J. Verbist et al., "Real-time and DSP-free 128 Gb/s PAM-4 link using a binary driven silicon photonic transmitter," J. Lightw. Technol., vol. 37, no. 2, pp. 274-280, 2019.

[13] H. Li et al., "A $112 \mathrm{~Gb} / \mathrm{s}$ PAM4 transmitter with silicon photonics microring modulator and CMOS driver," in Proc. Opt. Fiber Comm. Conf. Exhibit., 2019, p. Th4A.4.

[14] G. T. Reed, G. Mashanovich, F. Y. Gardes, and D. J. Thomson, "Silicon optical modulators," Nat. Photon., vol. 4, no. 8, pp. 518-526, 2010.

[15] J. Witzens, "High-speed silicon photonics modulators," Proc. IEEE, vol. 106, no. 12, pp. 2158-2182, 2018.

[16] R. Soref and B. Bennett, "Electrooptical effects in silicon," IEEE J. Quantum Electron., vol. QE-23, no. 1, pp. 123-129, Jan. 1987.

[17] G. L. Li, T. G. B. Mason, and P. K. L. Yu, "Analysis of segmented traveling-wave optical modulators," J. Lightw. Technol., vol. 22, no. 7, pp. 1789-1796, 2004.

[18] L. Breyne et al., "Electro-optic frequency response shaping in high speed Mach-Zehnder modulators," in Proc. Opt. Fiber Commun. Conf. Exhib., 2020, Paper Th2A.11.

[19] D. Patel, M. Parvizi, N. Ben-Hamida, C. Rolland, and D. V. Plant, "Frequency response of dual-drive silicon photonic modulators with coupling between electrodes," Opt. Express, vol. 26, no. 7, pp. 8904-8915, Apr. 2018.

[20] L. Breyne et al., "50 GBd PAM4 transmitter with a 55 nm SiGe BiCMOS driver and silicon photonic segmented MZM," Opt. Express, vol. 28, no. 16, pp. 23 950-23 960, Aug. 2020.

[21] D. M. Pozar, Microwave Engineering, Fourth Edition ed. Hoboken, NJ, USA: Wiley, 2011.

[22] J. G. Proakis and D. K. Manolakis, Digital Signal Process., Fourth Edition ed. Essex, U.K.: Pearson, 2014.

[23] W. Bogaerts, P. Dumon, D. V. Thourhout, and R. Baets, "Low-loss, lowcross-talk crossings for silicon-on-insulator nanophotonic waveguides," Opt. Lett., vol. 32, no. 19, pp. 2801-2803, Oct. 2007.

[24] P. Absil et al., "Reliable $50 \mathrm{~Gb} / \mathrm{s}$ silicon photonics platform for nextgeneration data center optical interconnects," in Proc. IEEE Int. Electron. Devices Meeting , 2017, pp. 34.2.1-34.2.4.

[25] J. Wang and K. Petermann, "Small signal analysis for dispersive optical fiber communication systems," J. Lightw. Technol., vol. 10, no. 1, pp. 96-100, 1992. 